\title{
IMPLANTABLE 3D PRINTED DRUG DELIVERY SYSTEM
}

\author{
Khalil Moussi ${ }^{*}$, Abdullah Bukhamsin, and Jurgen Kosel \\ Computer, Electrical and Mathematical Sciences and Engineering Division \\ King Abdullah University of Science and Technology, Thuwal, Saudi Arabia
}

\begin{abstract}
A miniaturized drug delivery system suitable for invivo biomedical applications is presented. The system consists of an electrolytic pump driving a micro bellows membrane as an actuator for delivery through microneedles. A two-photon polymerization $3 \mathrm{D}$ printing technique was used to fabricate a reservoir equipped with microneedles. Analytical characterization of the flow rate through the microneedles showed an outgoing flow rate ranging from $63 \mu \mathrm{L} / \mathrm{min}$ to $520 \mu \mathrm{L} / \mathrm{min}$ for an applied pressure of 0.1 to $1 \mathrm{kPa}$. The assembled system with an overall size of $3.9 \mathrm{~mm} \times 2.1 \mathrm{~mm} \times 2 \mathrm{~mm}$ achieved delivery of $4 \pm 0.5 \mu \mathrm{L}$ within 12 seconds of actuation. A penetration test of the microneedle into a skin-like material confirms its potential for transdermal delivery.
\end{abstract}

\section{KEYWORDS}

3D printing, drug delivery, electrolytic micropump, microneedles.

\section{INTRODUCTION}

Conventional drug delivery routes provide limited control over the spatial and temporal resolution of drug release. Often, the desired availability of the therapeutic drug in the target site can only be achieved by either increasing the dose volume or the dosing frequency, both of which are undesired, due to side effects and low patient compliance [1]. One way to circumvent this issue is via direct injection of drugs into the target site. This strategy, however, cannot be used to reach remote areas of the body and has to be done repeatedly to achieve the desired therapeutic effect, leading to trauma and risk of infections [2]. As such, alternative approaches to drug administration have been intensely investigated.

One such approach is the use of coatings that confer selectivity to a generic drug. The drug can be coated with polymers, nanoparticles, liposome, or specific cellreceptor ligands that allow the drug to evade being systematically cleared out by the body and accumulate at the desired target area. The drug cargo may then be released using an external stimulus, such as localized heating, or environment sensing mechanisms, such as $\mathrm{pH}-$ sensitive hydrolyzing polymers [3]. Microparticles or nanoparticles can be exploited similarly to carry and release drugs [4]. While this approach may allow for selective targeting, it does confer no or little control over the release rate of the drug.

These issues have fueled interest in the use of biocompatible and miniaturized delivery platforms that can be implanted using minimally invasive procedures. Such platforms allow for a controlled and targeted release of drugs by using actuators that are coupled to a drug reservoir. Osmotic actuators have been very popular, but provide no or limited control of the delivery rate [5]. Electrolytic actuators have been gaining traction being implemented into drug delivery platforms, due to their simplicity and efficiency [6]. Conventional electrolytic actuators utilize a diaphragm-design, in which the electrolysis of water drives the deflection of the diaphragm. This deflection pushes the drug from an adjacent reservoir compartment through a funneled cannula to the target site. In this configuration, the release rate can be easily controlled by limiting the supplied current driving the electrolysis reaction. Versatile delivery systems with attractive features including wireless operation and valve control have been developed, but integration into a compact package is lacking [7].

In this study, we propose integrating a miniaturized electrolytic pump that utilizes a flexible Parylene $\mathrm{C}$ bellows membrane and a reservoir with microneedles (MNs) for direct injection into the desired target site. The customizable bellows and reservoir dimensions allow achieving delivery of volumes in the range of $1 \mu \mathrm{L}$ to 20 $\mu \mathrm{L}$, which is adequate for the treatment of different diseases such as tumors [8]. A fluidic characterization of the reservoir and $\mathrm{MNs}$ is presented. In addition, a characterization of the electrolysis micropump flow rate and efficiency was achieved. We also assess the penetration capability of a $500 \mu \mathrm{m}$ long $\mathrm{MN}$ through a $390 \mu \mathrm{m}$ thick polydimethylsiloxane (PDMS) film.

\section{CONCEPT AND DESIGN}

As shown in Fig. 1, the delivery system is composed of an electrolytic micropump and a microneedlesequipped reservoir $(3.9 \mathrm{~mm} \times 2.1 \mathrm{~mm} \times 2 \mathrm{~mm})$. The micropump can be powered using a DC voltage supply or wirelessly using an inductive powering unit. The electrolytic micropump consists of interdigitated electrodes and a Parylene C micro-bellows membrane [9].

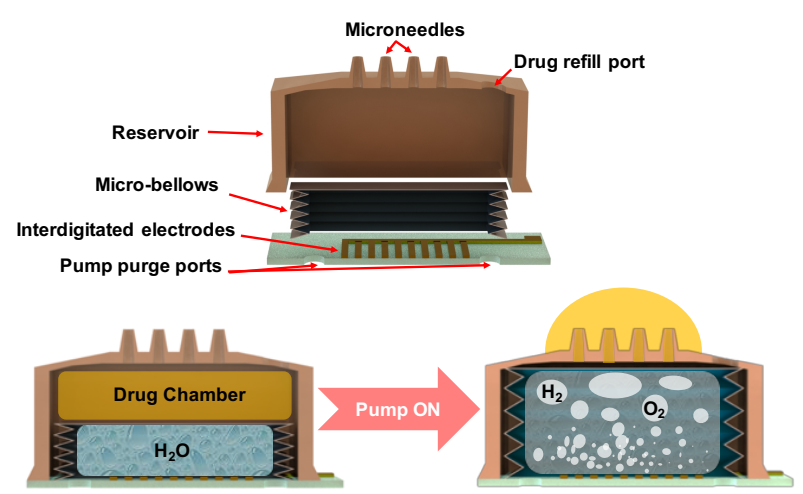

Figure 1: Schematic diagram of the drug delivery system and illustration of the operation. 
When applying a constant current to the interdigitated electrodes, water electrolysis initiates generating oxygen/ hydrogen bubbles, which induce a volume increase expanding the bellows membrane. Therefore, the Parylene $\mathrm{C}$ membrane pushes the drug amount through the microneedles to the targeted site.

\section{FABRICATION}

Figure 2 shows the 3D printing process for the fabrication of a reservoir $(3.9 \mathrm{~mm} \times 2.1 \mathrm{~mm} \times 2 \mathrm{~mm})$ with microneedles diameter and height of $100 \mu \mathrm{m}$ and $300 \mu \mathrm{m}$, respectively) using the Nanoscribe Photonic Professional GT laser lithography system (Nanoscribe $\mathrm{GmbH}$, Germany).

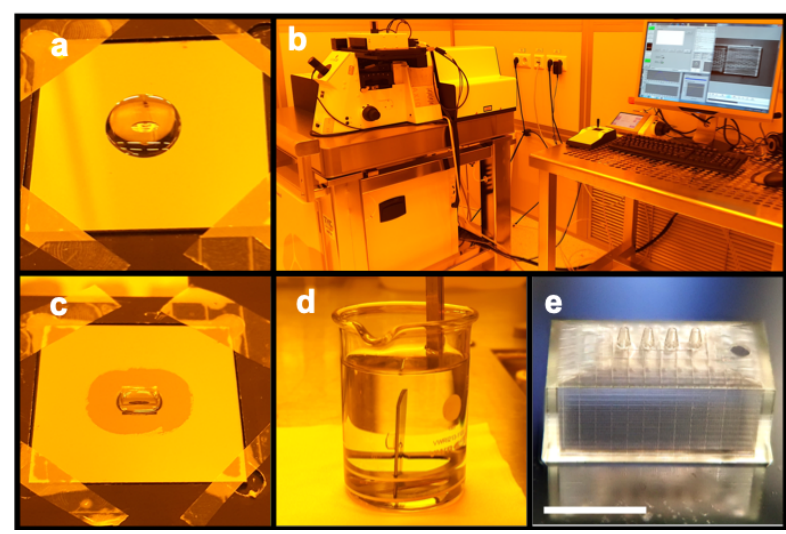

Figure 2: (a-d) Fabrication process of the 3D printed reservoir with microneedles; (e) reservoir with $4 \mathrm{MNs}$ (100 $\mu \mathrm{m}$ diameter, $300 \mu \mathrm{m}$ long).

A $25 \times 25 \mathrm{~mm}^{2}$ silicon substrate with a hole $(0.9 \mathrm{~mm}$ in diameter) in the center was used for $3 \mathrm{D}$ printing. The hole helps cleaning the unpolymerized resist in the development step. After drop-casting IP-S photoresist (Nanoscribe GmbH, Germany), the sample is then loaded and $3 \mathrm{D}$ printing starts (Figure 2a-b). The printing scan speed and power were $50 \mathrm{~mm} / \mathrm{s}$ and $120 \mathrm{~mW}$ respectively, and the laser wavelength is $780 \mathrm{~nm}$. Then, the sample was immersed for 10 minutes in mr-DEV 600 developer followed by 10 minutes in isopropanol (Figure 2c-d).

Interdigitated electrodes $(20 \mathrm{~nm} / 200 \mathrm{~nm} \mathrm{Ti} / \mathrm{Pt}$ with $100 \mu \mathrm{m}$ width by $100 \mu \mathrm{m}$ spacing) were fabricated on silicon and silicon dioxide substrates by a liftoff process.

Parylene $\mathrm{C}$ microbellows membrane was fabricated by a double mold-casting process previously reported [6].

\section{EXPERIMENTS AND RESULTS}

\section{Microneedle fluidic characterization}

The total flow outgoing from the reservoir through the microneedles has been determined using the Extended Bernoulli's Equation (EBE) (Eq. 1) for an incompressible fluid having losses between two points; the first point represents the entrance of the reservoir where a presser $p_{1}$ is applied, while, the second point is at the exit of the MNs under atmospheric pressure $\mathrm{p}_{2}$ as shown in figure 2 .

$$
\mathrm{p}_{1}-\mathrm{p}_{2}+\frac{\rho\left(\mathrm{V}_{1}^{2}-\mathrm{V}_{2}^{2}\right)}{2}+\rho g\left(\mathrm{z}_{1}-\mathrm{z}_{2}\right)=\sum_{\mathrm{i}=1}^{4} \mathrm{p}_{\text {loss } \mathrm{i}}
$$

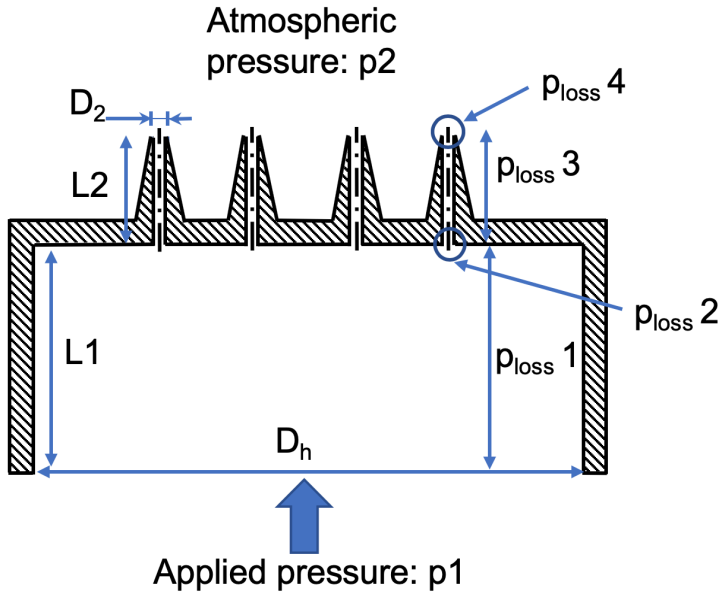

Figure 3: Simplified diagram of the fluidic system reservoir and microneedles.

As the reservoir and MNs were tested in a horizontal orientation $\left(\mathrm{Z}_{1}=\mathrm{Z}_{2}\right)$, the gravity term in Eq. 1 does not affect the fluidic system. In every piping systems, there exist pressure losses divided into two main types. On the one hand, the major losses are due to the friction between the fluid and the inner peripheral surface of the pipe

$$
\mathrm{p}_{\text {loss } \mathrm{i}}=\mathrm{f}_{\mathrm{i}}\left(\mathrm{L}_{\mathrm{i}} / \mathrm{D}_{\mathrm{i}}\right) \rho\left(\mathrm{V}_{\mathrm{i}}^{2} / 2\right) \quad(\mathrm{i}=1,3)
$$

where $f$ is the Darcy friction factor, which can be expressed for a laminar flow as

$$
\mathrm{f}=64 / \mathrm{R}_{\mathrm{e}}
$$

In Eq. (3) $R_{\mathrm{e}}$ is the Reynolds number and defined as

$$
\mathrm{R}_{\mathrm{e}}=\rho v_{\mathrm{avg}} \mathrm{D} / \mu
$$

On the other hand, minor losses are caused by the change of the flow velocity due to pipe bends, enlargements, contractions, valves, etc.

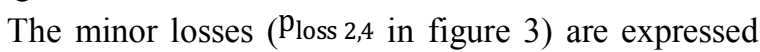
in terms of the loss coefficient $\mathrm{K}_{\mathrm{i}}$ which represents, in this case, the losses at the entrance (a sharp-edged inlet $\mathrm{K}=0.5)$ and the $\mathrm{MNs}$ outlet $(\mathrm{K}=1)(\mathrm{Eq} .5)$.

$$
p_{\text {loss } i}=K_{i} \rho\left(V_{i}^{2} / 2\right) \quad(i=2,4)
$$

The law of continuity for an incompressible fluid states that the product of the average velocity Vavg and the cross-sectional pipe area A must remain constant for a given stream flow. Applying this law to the fluidic system in Fig. 3 and assuming an equal repartition of the volumetric flow between the MNs, the continuity law between the points (1) and (2) can be expressed as

$$
\mathrm{v}_{\text {avg } 1} \mathrm{~A}_{1}=\mathrm{N} \text { vavg2} \mathrm{A}_{2}
$$

Where $\mathrm{N}$ is the number of MNs.

Substituting Eqs. (2), (3), (4), (5), and (6) into Eq. (1), the flow velocity in a single $\mathrm{MN}$ was calculated. Then, the total flow rate through the MNs was determined by

$$
\mathrm{Q}_{\mathrm{v}}=\mathrm{Nv}_{\mathrm{avg} 2} \mathrm{~S}_{2}
$$


Figure 4 shows a fluidic characterization of the flow rate through $\mathrm{MNs}$ at an applied pressure ranging from 0.1 to $1 \mathrm{kPa}$ where the achievable flow rate ranged from 63 $\mu \mathrm{L} / \mathrm{min}$ to $520 \mu \mathrm{L} / \mathrm{min}$.

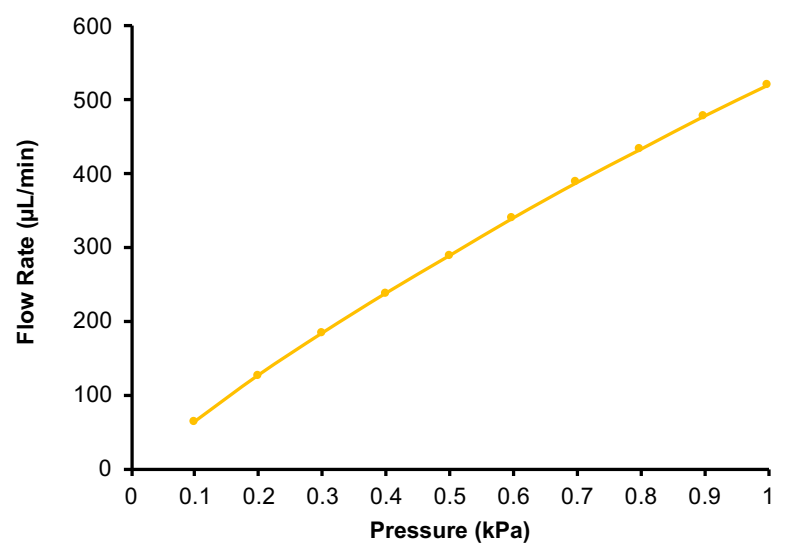

Figure 4: Flow rate versus pressure

\section{Electrolytic pump}

To measure the flow rates that can be generated by the electrolytic pump, a PMMA chamber with two horizontal channels was fixed on top of the electrodes (Fig. 5). One channel was used to fill the chamber with DI water and then sealed during functional testing to prevent backflow. The second channel was connected to $15 \mathrm{~cm}$ of tubing that lay in parallel to a $0.5 \mathrm{~mm}$ graduated ruler to quantify fluid displacement. A current supply was used to test the system from $0.2 \mathrm{~mA}$ up to $1 \mathrm{~mA}$.

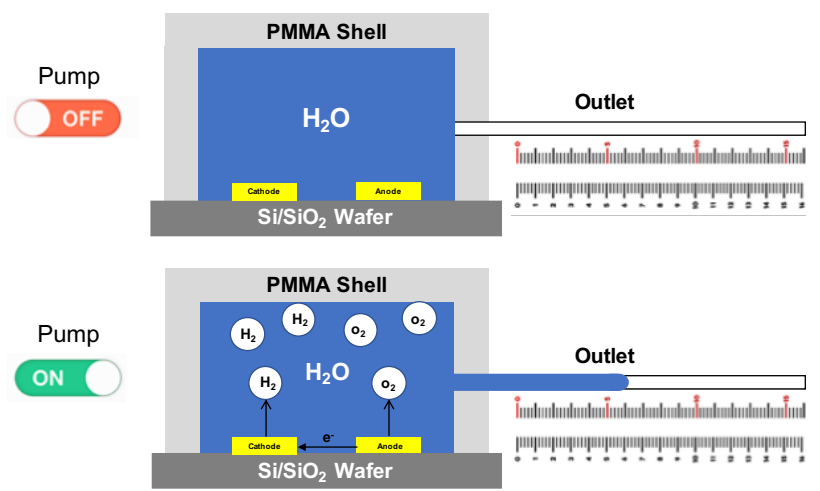

Figure 5: Electrolysis pump characterization setup.

Using the time duration of the test and its respective fluid displacement, the flow rate of the system was calculated. Electrodes were patterned on both $\mathrm{Si}$ and $\mathrm{SiO}_{2}$ substrates to determine if the passivation layer of $\mathrm{SiO}_{2}$ has any impact on the efficiency of the electrodes. As can be seen in Figure 6, flow rates as low as $0.08 \mu \mathrm{L} / \mathrm{min}$ at $0.2 \mathrm{~mA}$ and as high as $8.8 \mu \mathrm{L} / \mathrm{min}$ at $1 \mathrm{~mA}$ were achieved. The $\mathrm{SiO}_{2}$ passivation increased the flow rate for all the current values. This is caused by the current leakage between the electrodes through the $\mathrm{Si}$ substrate without using a $\mathrm{SiO}_{2}$ passivation.

The efficiency of the electrodes was calculated based on a ratio of the experimentally determined volume to the theoretical volume.
The theoretical volume $\left(\mathrm{V}_{\text {Theoretical }}\left(\mathrm{m}^{3}\right)\right)$ is given by

$$
\mathrm{V}_{\text {theortical }}=\left(\frac{3}{4} * \frac{\mathrm{I}}{\mathrm{F}} * \mathrm{~V}_{\mathrm{M}}\right) * \text { time }
$$

Where $\mathrm{I}$ is the current (in $\mathrm{A}$ ), $\mathrm{V}_{\mathrm{M}}$ is the molar gas volume at standard conditions $\left(24.7 \times 10^{-3} \mathrm{~m}^{3} / \mathrm{mol}\right), \mathrm{F}$ is Faraday's constant $\left(96.49 \times 10^{3} \mathrm{C} / \mathrm{mol}\right)$, and the time is in seconds.

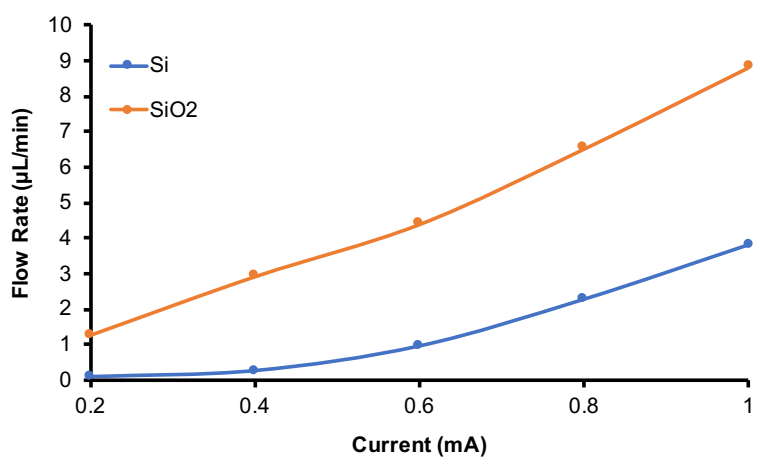

Figure 6: Flow rate results for silicon and silicon dioxide substrates.

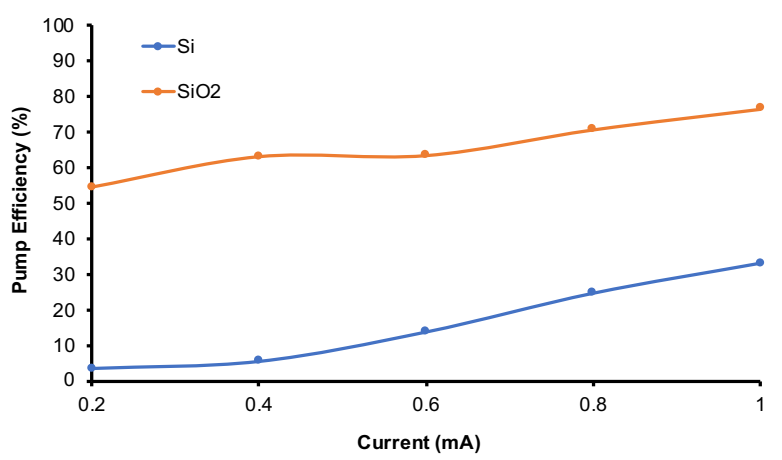

Figure 7: Pump efficiency for silicon and silicon dioxide substrates.

The efficiency of the electrodes ranged from $3.5 \%$ at 0.2 $\mathrm{mA}$ to $32.9 \%$ at $1 \mathrm{~mA}$ for $\mathrm{Si}$ and from $54.5 \%$ at $0.2 \mathrm{~mA}$ to $76.4 \%$ at $1 \mathrm{~mA}$ for $\mathrm{SiO}_{2}$ with a power consumption varying from $0.6 \mathrm{~mW}$ to $21 \mathrm{~mW}$. The results show that the efficiency of the electrodes increased with increasing currents for both $\mathrm{Si}$ and $\mathrm{SiO}_{2}$ substrate.

\section{Microneedles insertion test}

Figure 8a shows the applied force as a function of the MN displacement against a skin-like material (PDMS layer 10:1 base/curing agent), where puncturing happened at $95 \mathrm{mN}$. Figure $8 \mathrm{~b}$ shows a scanning electron microscopy images of the $\mathrm{MN}$ tip going through the PDMS film. The MN appears undamaged, confirming sufficient mechanical stability.

\section{Assembly and test of the drug delivery system}

The integrated system was validated by testing the full assembly. The reservoir was filled with red dye to simulate the drug delivery during the experiment as shown in Fig. 9. This depicts the delivery of $\sim 4 \mu \mathrm{L}$ red dye through the MNs during the first 12 seconds of actuation using a DC voltage supplier $(21 \mathrm{~V}, 2 \mathrm{~mA})$. 
(a)

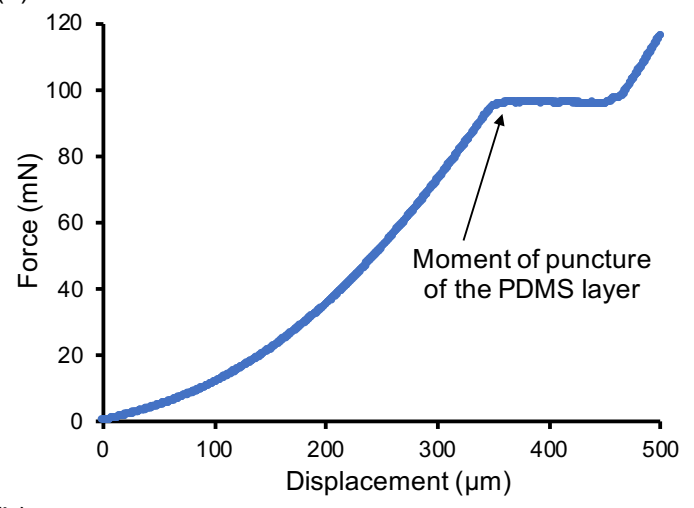

(b)

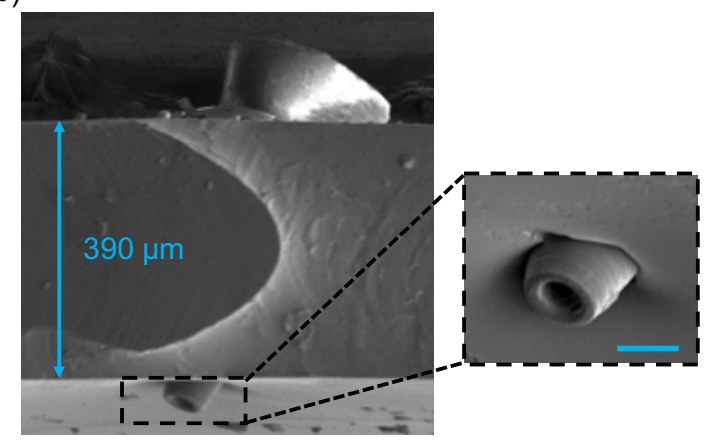

Figure 8: Penetration test of the $500 \mu \mathrm{m}$ long $M N$ into PDMS film; (a) Measurement of the insertion force as function of the MN displacement; (b) Scanning electron microscopy images of the MN penetration through a $390 \mu \mathrm{m}$ thick PDMS film. Scale bar $50 \mu \mathrm{m}$.

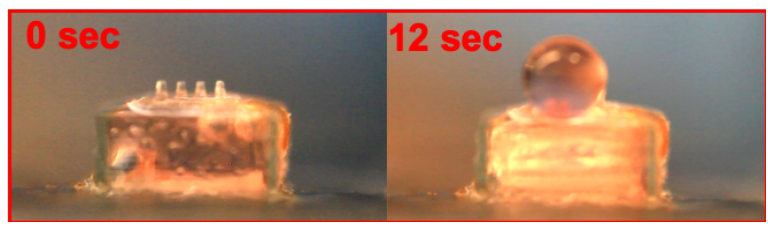

Figure 9: Photographs of the delivery system and red dye delivery within 12 seconds of actuation.

\section{CONCLUSION}

A versatile drug delivery system has been developed that, despite its miniaturization, provides a high control over delivery. The integrated electrolytic pump reaches an efficiency of $76 \%$, is capable of delivering the needed volumes for targeting tumors, and provides low-power operation. Coupled with a refillable reservoir, its miniaturized scale lends itself to long-term implantation for targeted drug delivery, where conventional methods are ineffective or suffer low patient-compliance, such as ocular injections.
The MNs fitted on the reservoir may also be used for transdermal delivery with the rate of delivery being controlled by the electrolytic pump.

\section{ACKNOWLEDGMENTS}

This work was funded and supported by King Abdullah University of Science and Technology (KAUST).

\section{REFERENCES}

[1] N. Elman and U. Upadhyay, "Medical applications of implantable drug delivery microdevices based on MEMS (Micro-Electro-Mechanical-Systems)," Current pharmaceutical biotechnology, vol. 11, no. 4, pp. 398-403, 2010.

[2] J. Ambati et al., "Transscleral delivery of bioactive protein to the choroid and retina," Investigative ophthalmology \& visual science, vol. 41, no. 5, pp. 1186-1191, 2000.

[3] D. Schmaljohann, "Thermo-and pH-responsive polymers in drug delivery," Advanced drug delivery reviews, vol. 58, no. 15, pp. 1655-1670, 2006.

[4] O. Yassine, A. Zaher, E. Li, E.A. Qiang, Alfadhel, J.E. Perez, M. Kavaldzhiev, M.F. Contreras, S. Li, S. T. Thoroddsen, N. M. Khashab, and J. Kosel, "Highly Efficient Thermoresponsive Nanocomposite for Controlled Release Applications," Scientific Reports, vol. 6, p. 28539, 2016.

[5] A. Zaher, S. Li, O. Yassine, N. Khashab, N. Pirmoradi, L. Lin, J. Kosel: "Osmotically driven drug delivery through remote-controlled magnetic nanocomposite membranes". Biomicrofluidics, vol. 9, no. 5 , p. $054113,2015$.

[6] P. Song, D. J. H. Tng, R. Hu, G. Lin, E. Meng, and K. T. Yong, "An electrochemically actuated MEMS device for individualized drug delivery: an in vitro study," Advanced healthcare materials, vol. 2, no. 8, pp. 1170-1178, 2013.

[7] Y. Yi, A. Zaher, O. Yassine, J. Kosel, I.G. Foulds, “A remotely operated drug delivery system with an electrolytic pump and a thermoresponsive valve," Biomicrofluidics, vol. 9, no. 5, p. 052608, 2015.

[8] W. D. Paulson et al., "Safety and efficacy of local periadventitial delivery of sirolimus for improving hemodialysis graft patency: first human experience with a sirolimus-eluting collagen membrane (CollR)," Nephrology Dialysis Transplantation, vol. 27, no. 3, pp. 1219-1224, 2012.

[9] K. Moussi and J. Kosel, "3-D Printed Biocompatible Micro-Bellows Membranes," J. Microelectromech. Syst., vol.27, no. 3, pp. 472-478, 2018.

\section{CONTACT}

*Khalil Moussi, khalil.moussi@kaust.edu.sa 